\title{
Identical Molecular Strings Woven Differently by Intermolecular Interactions in Dimorphs of myo-inositol 1,3,5-orthobenzoate.
}

\author{
Gaurav Bhosekar, ${ }^{\dagger}$ Chebrolu Murali, ${ }^{\ddagger}$ Rajesh G. Gonnade, ${ }^{\dagger}$ Mysore S. Shashidhar, ${ }^{* \neq}$ \\ Mohan M. Bhadbhade ${ }^{* \dagger}$
}

${ }^{\dagger}$ Center for Materials Characterization and Division of Organic Synthesis, National Chemical Laboratory, Pune, INDIA.

Supplementary Information

TG/DTA Analysis. The thermogravimatic analysis was carried out on Sieko make TG/DTA 32 with heating rate $10{ }^{\circ} \mathrm{C}$ per minute.

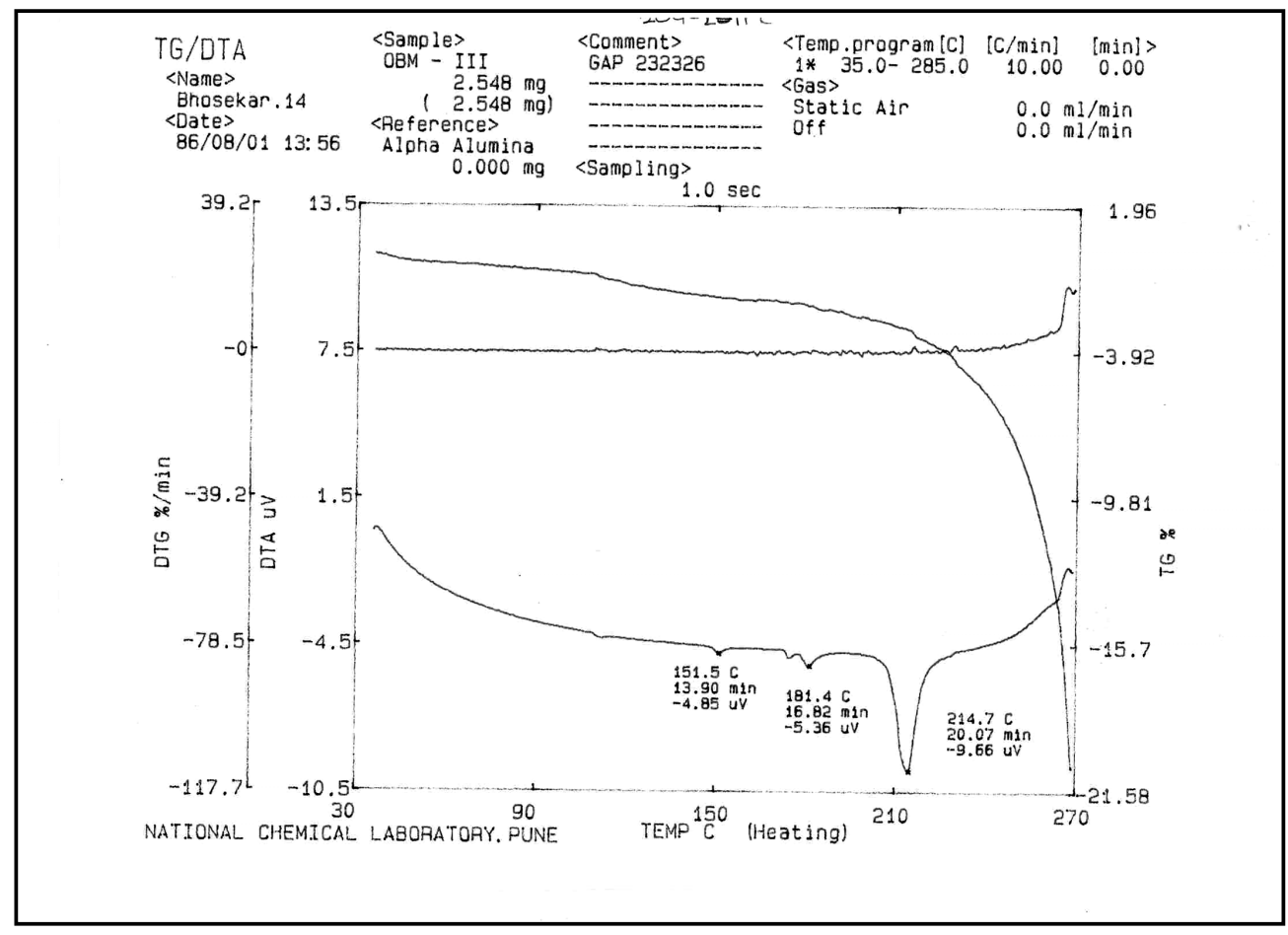

Fig. S1. TG / DTA data for Form I 


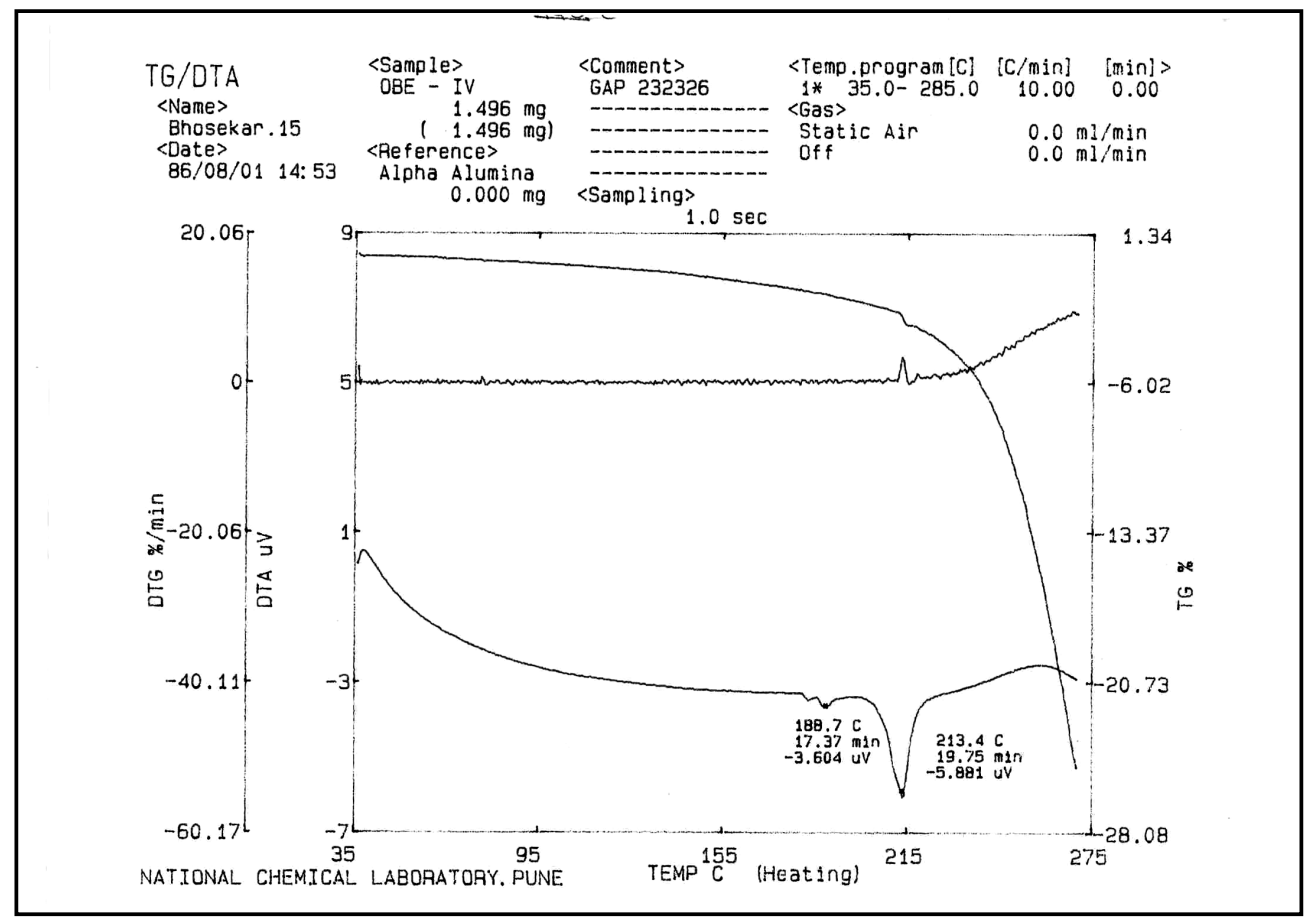

Fig. S2. TG / DTA data for Form II 